\title{
MEDIDAS DE EFECTO EN MEDICINA
}

\section{EFFECT MEASURES IN MEDICINE}

\section{Iván BARRIOS ${ }^{1}$, Roberto PÁEZ ${ }^{2}$.}

${ }^{1}$ Universidad Nacional de Asunción, Facultad de Ciencias Médicas, San Lorenzo, Paraguay.

${ }^{2}$ Universidad Nacional de Asunción, Facultad de Ciencias Exactas y Naturales, San Lorenzo, Paraguay.

Cómo citar este artículo: Barrios I, Páez R. Medidas de efecto en medicina. Med. clín. soc. 2019;3(2):68-70.

\section{Estimado Editor,}

El motivo de la misiva es compartir algunos conceptos clave sobre las medidas de efecto que se pueden utilizar en bioestadística, puesto que muchas veces no se utilizan o se utilizan de forma equivocada, siendo que son medidas fáciles de calcular e interpretar si es que se cumplen todos los supuestos metodológicos. Se hablará del odds ratio, el riesgo relativo y el riesgo absoluto.

En los estudios clínicos, el concepto de asociación se refiere a la existencia de un vínculo de dependencia entre una variable y otra. En general, la forma de identificar la asociación es a través de la comparación de dos o más grupos, para determinar si la frecuencia, magnitud o la presencia de una de las variables modifica la frecuencia de la otra en algún sentido. Sin embargo, hay que tener claro que esta asociación no implica una relación de causa - efecto (1).

El odds ratio es una medida típicamente asociada a los estudios de casos y controles, el odds de exposición es la probabilidad de haber estado expuesto dividida entre la probabilidad de no haber estado expuesto:

$$
\text { odds }=\frac{\text { Probabilidad de exposición }}{\text { Probabilidad de no exposición }}
$$

El cociente entre el odds entre casos (con la enfermedad o fenómeno estudiado) y controles (sin la enfermedad o fenómeno estudiado) se conoce como odds ratio (OR):

$$
\text { odds ratio }=\frac{\text { Odds de un caso a ser expuesto }}{\text { Odds de un control a ser expuesto }}
$$

Cuando no hay efecto el resultado será próximo a 1 y cuanto mayor sea el efecto más se alejará de él, en caso de que el OR sea menor que 1 se puede pensar que la variable estudiada es protectora. EI OR no es una medida de riesgo, es una medida de fuerza de asociación. Se debe presentar con su intervalo de confianza, ya que, si dentro del intervalo se encuentra el 1 , el OR no es estadísticamente significativo (2). 
Cabe mencionar que la medida se calcula a partir de los datos de una muestra y que se utiliza para estimar los grados de probabilidad de la población y por ello la estimación por intervalos es importante para cubrir un porcentaje de confianza de que en el rango estimado se encuentre el verdadero valor del OR. La fórmula del Intervalo de confianza del OR es:

$$
100(1-\alpha) \% C I=\widehat{O R}^{1 \pm\left(z_{\alpha} / \sqrt{\chi^{2}}\right)}
$$

Pero, ¿por qué se dice que el OR no es una medida de riesgo?, se define riesgo como la probabilidad de que un individuo desarrolle una enfermedad o presente otro desenlace en un período de tiempo dado (1), por lo tanto, es una característica que se mide a lo largo del tiempo, como los OR son característicos de los estudios de casos y controles, y estos a su vez son principalmente retrospectivos (aunque pueden ser prospectivos) no es una medida que pueda indicar riesgo. Existe una excepción a esto, y es cuando tanto la prevalencia y la incidencia de la enfermedad son bajas y el OR convergen con las medidas de riesgo relativo (3).

El riesgo relativo (RR) es una relación de la probabilidad de que ocurra un evento en el grupo expuesto versus la probabilidad de que ocurra en el grupo no expuesto. Un ejemplo es el RR de desarrollar cáncer de pulmón (evento) en fumadores (grupo expuesto) versus no fumadores (grupo no expuesto). El RR no proporciona ninguna información sobre el riesgo absoluto de que ocurra el evento, sino más bien la probabilidad más alta o más baja del evento en la exposición versus el grupo sin exposición (4).

$$
\text { Riesgo relativo }=\frac{\text { Incidencia de la enfermedad en expuestos }}{\text { Incidencia de la enfermedad en no expuestos }}
$$

El RR considera que el hecho de que algunas enfermedades ocurren en individuos no expuestos (como los no fumadores que tienen cáncer de pulmón), refleja el aumento en la cantidad de enfermedad observada en individuos expuestos, el RR es una medida de fuerza de asociación. La interpretación del RR es similar a la del OR, si el RR está próximo a 1, no hay ninguna asociación entre exposición y desarrollo de la enfermedad (2).

El riesgo absoluto (RA) es el riesgo histórico en individuos no expuestos. El riesgo también puede expresarse en términos absolutos mediante la diferencia de riesgo absoluto (conocido también como riesgo atribuible o diferencia de riesgo). Esta medida de efecto absoluta representa la diferencia entre los riesgos en dos grupos; generalmente entre un grupo expuesto y otro no expuesto. La diferencia de riesgo (DR), es la forma más útil de presentar los resultados de la investigación para ayudar a tomar decisiones (5).

Riesgo atribuible = riesgo en el grupo expuesto - riesgo en el grupo no expuesto

La DR se puede utilizar para calcular el número necesario para tratar (NNT), que es una medida relevante en la evaluación de la efectividad de una intervención de atención médica, generalmente un tratamiento con medicamentos. EI NNT es el inverso de la diferencia de riesgo absoluto y, por lo tanto, simplemente se puede calcular dividiendo 1 por la DR (5). Nos da una idea de cuántos individuos tendrán que ser expuestos al tratamiento para poder recoger algún beneficio en un solo individuo. 
En la tabla 1 se puede observar un resumen tomado de la publicación de Kim sobre las principales medidas de efecto utilizadas en medicina (6).

\begin{tabular}{|l|c|c|c|}
\hline \multicolumn{4}{|l|}{ TABLA 1. EJEMPLO DE TABLA CRUZADA 2 X2 CON RESUMEN DE LAS FÓRMULAS } \\
\hline & Expuesto & No expuesto & Total \\
\hline Enfermo & $a$ & $b$ & $a+b$ \\
\hline No enfermo & $c$ & $d$ & $c+d$ \\
\hline Total & $a+c$ & $b+d$ & $a+b+c+d$ \\
\hline Riesgo (de enfermedad) & $a /(a+c)$ & $b /(b+d)$ & \\
\hline Odds & $a / c$ & $b / d$ & \\
\hline Diferencia de riesgo & & & $(a /[a+c])-(b /[b+d])$ \\
\hline Riesgo relativo & & & $(a /[a+c]) /(b /[b+d])$ \\
\hline Odds ratio & & & $(a / c) /(b / d)$ \\
\hline
\end{tabular}

\section{CONFLICTOS DE INTERÉS Y FUENTE DE FINANCIACIÓN}

Los autores declaran no poseer conflictos de interés. Fuente de financiación: ninguna.

\section{REFERENCIAS BIBLIOGRÁFICAS}

1. Araujo M. Concepto de asociación, causa y riesgo. Medwave. 2011;11(01):e4843. https://dx.doi.org/10.5867/medwave.2011.01.4843

2. Dawson GF. Interpretación fácil de la bioestadística: la conexión entre la evidencia y las decisiones médicas. Madrid: Elsevier; 2009.

3. Feng $\mathrm{C}$, Wang $\mathrm{B}$, Wang $\mathrm{H}$. The relations among three popular indices of risks. Stat Med. 2019;38(23):4772-87. https://dx.doi.org/10.1002/sim.8330

4. Tenny S, Hoffman MR. Relative Risk. StatPearls, Treasure Island (FL): StatPearls Publishing; 2019.

5. Noordzij M, van Diepen M, Caskey FC, Jager KJ. Relative risk versus absolute risk: one cannot be interpreted without the other. Nephrol Dial Transplant. 2017;32(suppl_2):ii13-8. https://dx.doi.org/10.1093/ndt/gfw465

6. Kim H-Y. Statistical notes for clinical researchers: Risk difference, risk ratio, and odds ratio. Restor Dent Endod. 2017;42(1):72-6. https://dx.doi.org/10.5395/rde.2017.42.1.72 